\title{
Radiological and hormonal improvements in a 22-year-old patient with lymphocytic hypophysitis - the watchful waiting approach
}

\author{
Aleksandra Gilis-Januszewska, Łukasz Kluczyński, Damian Rogoziński, Alicja Hubalewska-Dydejczyk \\ Chair and Department of Endocrinology, Jagiellonian University Medical College, Krakow, Poland
}

Key words: hypophysitis; pituitary insufficiency; hypopituitarism; diabetes insipidus; pituitary stalk lesion

\section{Case presentation}

Hypophysitis is a rare condition describing inflammation of the pituitary gland. The disorder may be primary, involving the pituitary gland itself, or secondary, due to other diseases or drugs. The incidence of primary hypophysitis is one case per 9 million [1]; however, new forms of the condition are increasingly diagnosed and the actual occurrence appears to be higher. Symptoms are connected with the presence of a pathological mass in the pituitary area and hormonal abnormalities. The diagnosis is based on clinical presentation, laboratory tests, and imaging. Deficiency of ACTH is found in $60 \%$ of cases, followed by deficits of $\mathrm{LH} / \mathrm{FSH}$ in $52 \%$, TSH in $52 \%, \mathrm{ADH}$ in $39 \%, \mathrm{GH}$ in $38 \%$, and a high PRL level in $37 \%$ of cases [2]. Magnetic resonance remains the gold standard for imaging. The homogenous enhancement of the pituitary gland, thickening of a non-deviated stalk, and a missing posterior lobe signal in a T1 modality are the most characteristic radiological changes [3]. Hormonal substitution is the cornerstone of therapy. In cases with severe neurological symptoms, high-dose steroids or other immunosuppressant drugs are used. Surgery and radiotherapy are options for patients with significant consequences of mass-effect and those resistant to pharmacological treatment. Hypophysitis often leads to atrophy of the pituitary gland. More than 70\% of patients require long-term hormonal substitution [4].

A 22-year-old patient without a significant past medical history was admitted to a regional hospital with a four-month history of excessive thirst and frequent urination. The occurrence of polydipsia and polyuria was associated with respiratory tract infection. The patient's hobbies included amateur kickboxing, but he denied any head trauma. Based on a water deprivation test, diabetes insipidus was diagnosed, with substantial improvement in symptom control after administration of desmopressin. MR of the pituitary area revealed a thickened non-deviated stalk of $4.5 \mathrm{~mm}$ and a missing posterior pituitary lobe signal. During several hospitalisations in the Endocrinology Department, gonadal and somatotropic axis insufficiencies as well as mild hyperprolactinaemia were discovered. Adequate control of diabetes insipidus was confirmed. Thyroid and adrenal gland function were unaffected. To exclude secondary causes of the observed symptoms, multiple imaging and biochemical studies were performed with unremarkable findings. The patient was diagnosed with lymphocytic hypophysitis. In a control MR the pituitary stalk was enlarged to $5 \times 5 \times 5 \mathrm{~mm}$ and the pituitary lobes did not show any deviations. Due to the lack of severe neurological symptoms, treatment was continued with desmopressin only. During a follow-up assessment, the stalk size normalised to $2.5 \times 3 \times 3 \mathrm{~mm}$ on MR (Fig. 1). Prolactin and testosterone values were within normal limits. Unfortunately, the diabetes insipidus was persistent, and only a transient improvement in IGF-1 levels was observed (Tab. 1).

This case report shows that diabetes insipidus may be the first manifestation of developing hypophysitis. The treatment should be individualised and tailored to each case. Patients presenting with mild symptoms can be approached with watchful waiting as a therapeutic option because hormonal and imaging improvements occur during the course of the disease [5]. Further research is needed to create algorithms for the management of hypophysitis.

\section{Conflict of interest}

None declared. 


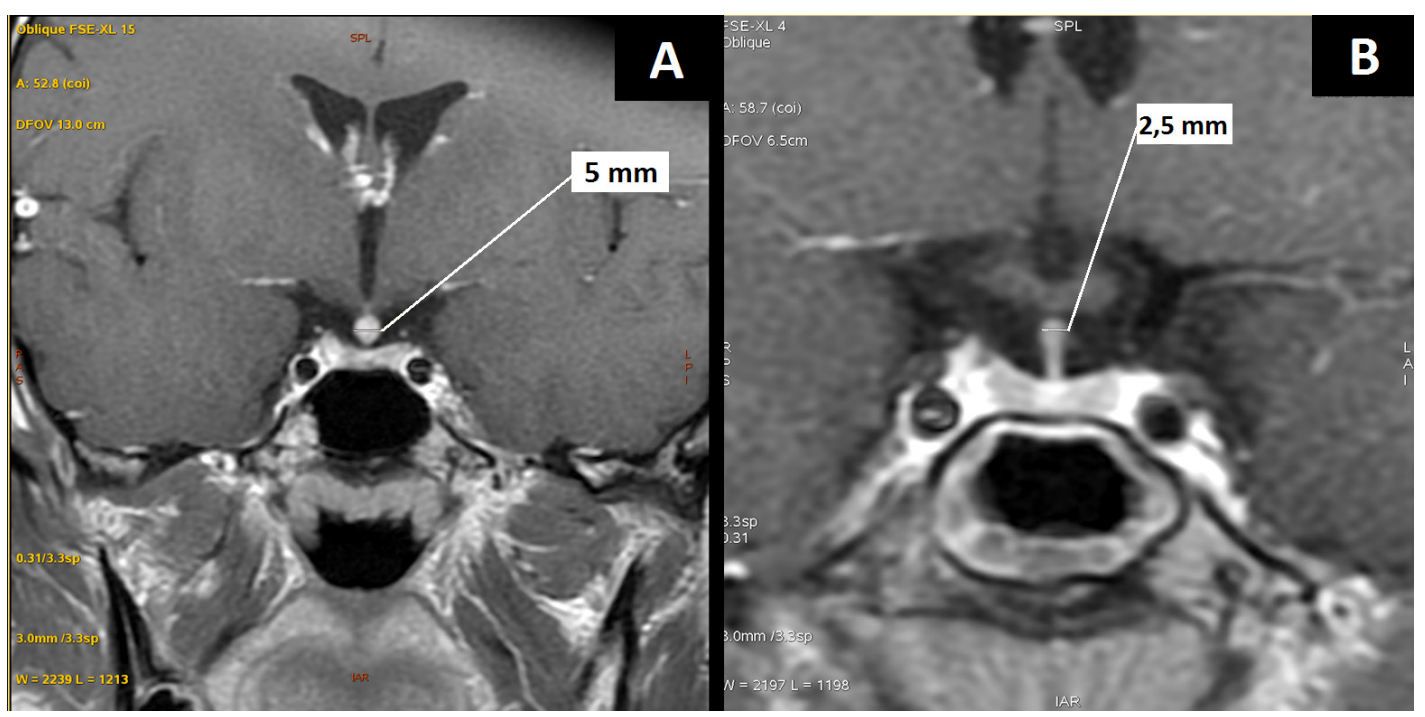

Figure 1. Radiological improvement in a 22-year-old patient with lymphocytic hypophysitis. Magnetic resonance imaging of the pituitary area. A. June 2018. B. January 2019. Courtesy of Department of Radiology, University Hospital, Krakow

Table 1. Hormonal assessment

\begin{tabular}{lccccc}
\hline Hormone [range] & IV 2018 & VI 2018 & IX 2018 & I 2019 & VI 2019 \\
\hline ACTH [6.0-56.0 pg/mL] & 42.0 & 41.6 & 45.0 & 55.0 & - \\
\hline Cortisol - morning & 11.6 & 22.1 & 18.8 & 19.3 & 15.1 \\
\hline TSH [0.27-4.20 ulU/mL] & 2.94 & 2.20 & 2.27 & 2.22 & 3.67 \\
\hline fT4 [12.0-22.0 pmol/L] & 13.4 & 12.5 & 13.5 & 16.6 & 16.65 \\
\hline PRL - morning [50.0-460.0 ulU/mL] & 384.0 & 792.4 & 263.0 & 302.1 & 311.7 \\
\hline LH [1.70-8.60 mlU/mL] & 4.07 & 4.33 & 4.75 & 5.18 & 5.34 \\
\hline FSH [1.5-12.4 mlU/mL] & 2.31 & 2.34 & 2.92 & 2.82 & 2.75 \\
\hline Testosterone [8.64-29.00 nmol/L] & 5.66 & 5.77 & 5.96 & 9.09 & 9.34 \\
\hline GH [0.2-10.0 ulU/mL] & 1,3 & 1.2 & 0.7 & 0.6 & - \\
\hline IGF-1 [235.0-408.0 ng/mL] & 225.0 & 164.0 & 265.0 & 253.0 & 215.0 \\
\hline Urine specific gravity [1.005-1.030 g/mL] & 1.016 & 1.021 & 1.017 & 1.011 & 1.003 \\
\hline
\end{tabular}

ACTH — adrenocorticotropic hormone; FSH — follicle-stimulating hormone; fT4 — free thyroxine; GH — growth hormone; IGF-1 — insulin-like growth factor 1; $\mathrm{LH}$ - luteinising hormone; PRL — prolactin; TSH — thyroid-stimulating hormone

\section{Authors contribution}

The first authorship of L.K. and A.G.J. is of equal rank.

\section{References}

1. Buxton N, Robertson I. Lymphocytic and granulocytic hypophysitis: a single centre experience. Br J Neurosurg. 2001; 15(3): 242-5, discussion 245, doi: 10.1080/02688690120057664, indexed in Pubmed: 11478060.

2. Prete A, Salvatori R. Hypophysitis. Endotext [Internet]. South Dartmouth 2018. https://www.ncbi.nlm.nih.gov/books/NBK519842/.
3. Bellastella G, Maiorino MI, Bizzarro A, et al. Revisitation of autoimmune hypophysitis: knowledge and uncertainties on pathophysiological and clinical aspects. Pituitary. 2016; 19(6): 625-642, doi: 10.1007/s11102-016-0736-z, indexed in Pubmed: 27503372.

4. Caturegli P, Newschaffer C, Olivi A, et al. Autoimmune hypophysitis. Endocr Rev. 2005; 26(5): 599-614, doi: 10.1210/er.2004-0011, indexed in Pubmed: 15634713

5. Kluczyński Ł, Gilis-Januszewska A, Rogoziński D, et al. Hypophysitis - new insights into diagnosis and treatment. Endokrynol Pol. 2019; 70(3): 260-269, doi: 10.5603/EP.a2019.0015, indexed in Pubmed: 31290557. 